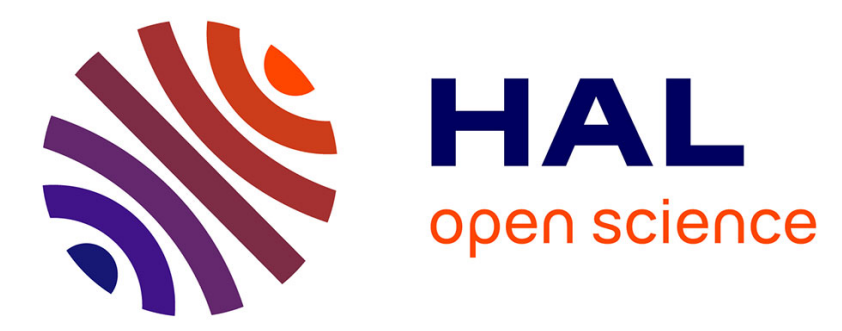

\title{
Finite-element analysis of errors on stress and strain measurements in dynamic tensile testing of low-ductile materials
}

\author{
Sana Koubaa, Ramzi Othman, Bassem Zouari, Sami El-Borgi
}

\section{- To cite this version:}

Sana Koubaa, Ramzi Othman, Bassem Zouari, Sami El-Borgi. Finite-element analysis of errors on stress and strain measurements in dynamic tensile testing of low-ductile materials. Computers \& Structures, 2011, 89 (1-2), pp.78-90. 10.1016/j.compstruc.2010.08.003 . hal-01006901

\section{HAL Id: hal-01006901 https://hal.science/hal-01006901}

Submitted on 5 May 2018

HAL is a multi-disciplinary open access archive for the deposit and dissemination of scientific research documents, whether they are published or not. The documents may come from teaching and research institutions in France or abroad, or from public or private research centers.
L'archive ouverte pluridisciplinaire HAL, est destinée au dépôt et à la diffusion de documents scientifiques de niveau recherche, publiés ou non, émanant des établissements d'enseignement et de recherche français ou étrangers, des laboratoires publics ou privés. 


\title{
Finite-element analysis of errors on stress and strain measurements in dynamic tensile testing of low-ductile materials
}

\author{
Sana Koubaa ${ }^{\mathrm{a}, \mathrm{b}}$, Ramzi Othman ${ }^{\mathrm{a}, \mathrm{c}, *}$, Bassem Zouari ${ }^{\mathrm{d}}$, Sami El-Borgi ${ }^{\mathrm{b}}$ \\ anstitut de Recherche en Génie Civil et Mécanique, Ecole Centrale de Nantes, 1 Rue de la Noë, BP 92101, 44321 Nantes cedex 3, France \\ ${ }^{\mathrm{b}}$ Applied Mechanics and Systems Research Laboratory, Tunisia Polytechnic School, BP 743, 2048 La Marsa, Tunisia \\ ' Structural Impact Laboratory (SIMLab), Centre for Research-based Innovation, Norwegian University of Science and Technology (NTNU), NO-7491 Trondheim, Norway \\ ${ }^{\mathrm{d}}$ UDSM, Ecole Nationale d'Ingénieurs de Sfax, Sfax, Tunisia
}

Tensile dynamic tests are essential experiments to develop and validate constitutive equations. In this paper, we studied the errors on stress and strain measurement in dynamic tensile tests by using finite-element analysis. Two strain and one stress measures were discussed. Mainly, we considered the influence of the material and multiple geometrical and testing parameters. We were limited to the case of elastic behaviour of the material. We observed that the errors on the strain measures are essentially influenced by the geometrical parameters. On the other hand, the error on the stress measure are highly correlated to stress field homogeneity.

\section{Introduction}

During past decades, the investigation of the dynamic material behaviour, at high strain rates, has become more and more prominent, in order to design stronger and more durable structures and components. Therefore, relevant constitutive equations that take into account strain rate sensitivity are a major concern in several engineering applications [1]. Consequently, numerous works studied the dynamic response of materials in compression [2-7], tension [6-16], shear [17-21] and multi-axial [22].

It is widely admitted that the specimen geometry can influence measurements in dynamic experiments. In other terms, the measured behaviour in these experiments is unavoidably the result of two contributions material and structural (sample geometry) effects. The structural contributions are in some cases as important as material response. Indeed, dynamic experimental techniques give force and displacement measurements at specimen boundaries. By means of some assumptions, we can deduce stress and strain in the specimen. Therefore, we obtain the stress-strain relation which should only be depended on the tested material. Actually, this is not true and the sample shape and dimensions may influence these stress-strain relations. Several works interested

\footnotetext{
* Corresponding author.

E-mail address: Ramzi.Othman@ec-nantes.fr (R. Othman).
}

in quantifying and correcting specimen geometry effects $[2,5,12,23-33]$.

The specimen geometry can yield many sub-effects such as inertia, wave propagation or stress concentration effects. It is therefore of much importance to wisely design samples in dynamic experiments. However, widely divergent specimen geometries and dimensions are used in literature. On the other hand, the majority of the cited references were only interested in specimen geometry effects in the case of dynamic compression tests [2,5,23-32]. Recently, Challita and Othman [34] investigated the case of doublelap bonded joints. Only Huh et al. [12] and Verleysen et al. [33] and Maringa [35] studied the case of dynamic tensile samples. In the last reference, this point is studied from an experimental point of view. They carried out tensile split Hopkinson tensile bar tests on TRIP steel with different geometries. They revealed that strain can be overestimated due to geometrical effects. However, this study was limited to the errors on strain measurement. Besides, Ref. $[12,35]$ approaches the geometry effects by numerical simulations. However, these works were not extensive and were only limited to the gauge zone.

In this paper, we set out to assess geometry effects on the stress and strain measurements in dynamic tensile experiments. Precisely, the sensitivity of errors to several geometrical, material and test parameters is investigated. Furthermore, we are interested in studying stress and strain homogeneity in the specimen. We consider only the case of low-ductile materials. Hence, only the materials behaviour will be assumed elastic. 


\section{Method}

\subsection{Reference model}

As we are interested in studying the effect of several geometrical, material and test parameters on the strain and stress measurement, we define a reference model. Subsequently, one parameter is changed a time.

The adopted geometry, in this work, is presented in Fig. 1. This choice is motivated by the fact that dogbone shape is the most used in tensile testing [33]. The specimen consists of five parts: central, two transition and two glue zones. The two glue zones are on both sides of the specimen. In real experiments, these glue zones are used to fix the specimen on the testing machines. In our numerical experiments, the glue zones are used to impose boundary conditions. The transition zones have a varying width. This zones length and width are characterised by the radius $\rho$. The central zone is the gauge section. In tensile experiments, we assume that the applied loading is integrally and instantanoeusly transmitted to the gauge section. Furthermore, we assume that glue and transition zones remain rigid and that only the central zone is deformed during the tensile test. The central zone has a length $l$ and a width $w$.

Following Ref. [33], we consider $l=5 \mathrm{~mm}, w=4 \mathrm{~mm}$ and $\rho=2 \mathrm{~mm}$. In addition, the sample thickness $t$ is taken $2 \mathrm{~mm}$ in the reference model. In all simulations, the glue zones are not considered and we assume that boundary conditions are directly applied to the glue-transition zones interfaces.

In order to quantify errors on stress and strain measurement in dynamic tensile experiments, numerical simulations are a powerful tool. The tensile sample is modeled by finite-element analysis using the commercial code ABAQUS 6.6-2. The adopted geometry is meshed with 2D-plan quadrilateral plane-stress elements. In the central and transition zones, the average mesh element length is $0.1 \mathrm{~mm}$ (Fig. 2). The left glue-transition zones interface is considered motionless. On the other hand, we impose the axial velocity profile presented in Fig. 3, at the right glue-transition zones interface. In this figure, $\tau_{r}$ and $\tau_{0}$ are the rise-time and total simulation time, respectively, and $V_{0}$ is the velocity amplitude. In the reference model, we consider $\tau_{r}=10 \mu \mathrm{s}$ and $V_{0}=10 \mathrm{~m} / \mathrm{s}$.

In this paper, we will consider an elastic behaviour of the material. Precisely, we consider an aluminum-like material, in the case of the reference model. Thus, the Young's modulus E, Poisson's ratio $v$ and density $d$ are taken equal to $70 \mathrm{GPa}, 0.34$ and $2800 \mathrm{~kg} / \mathrm{m}^{3}$, respectively. On the other hand, the total simulation time $\tau_{0}$ is taken as the time necessary to achieve an average axial strain in the central zone of $5 \%$.

\subsection{Analysis}

\subsubsection{Stress and strain measures}

In conventional dynamical tensile tests, the nominal stress is determined from a force measurement. This force is assumed to be the opposite of the reaction at the left glue zone. Let $\mathcal{R}^{A b}(\tau)$ be this reaction which is determined by the finite-element simulations on Abaqus. Hence, the force applied to the sample will be considered as: $\mathcal{F}^{A b}(\tau)$, reads:

$\mathcal{F}^{A b}(\tau)=-\mathcal{R}^{A b}(\tau)$

We should notice that $\mathcal{F}^{A b}$ is the experimental-like force measurement.

In order to obtain the stress measurement, we assume that the applied force is transmitted instantaneously to each cross-section of specimen, and particularly to cross-section of the central zone. Therefore, the nominal or engineering stress, in a cross-section of the central zone, is given by:

$\sigma_{n}(\tau)=\frac{\mathcal{F}^{A b}(\tau)}{S}=\frac{\mathcal{F}^{A b}(\tau)}{t \times w}$,

where $S$ holds for the cross-sectional area at the central zone. It is worth to recall that $w$ and $t$ are the width and thickness, respectively, of the central zone.

Furthermore, it is assumed that transition zones are rigid in order to obtain the strain. Only the central zone is supposed to deform. Moreover, the strain field is considered homogeneous in this zone. Therefore, the nominal or engineering strain is deduced from the displacement of the right glue zone as follows:

$\epsilon_{n}(\tau)=\frac{\mathcal{U}^{A b}(\tau)}{l}$

where $\mathcal{U}^{A b}(\tau)$ is the displacement of right glue zone and $l$ the central zone length. The displacement is determined from the imposed velocity profile given in Fig. 3. Here also, $\mathcal{U}^{A b}(\tau)$ is considered as the experimental-like displacement measurement.

The assumption of rigid transition zones may be not valid, mainly, when the radius of these zones has the same order of magnitude then the specimen length. If we take into account the whole

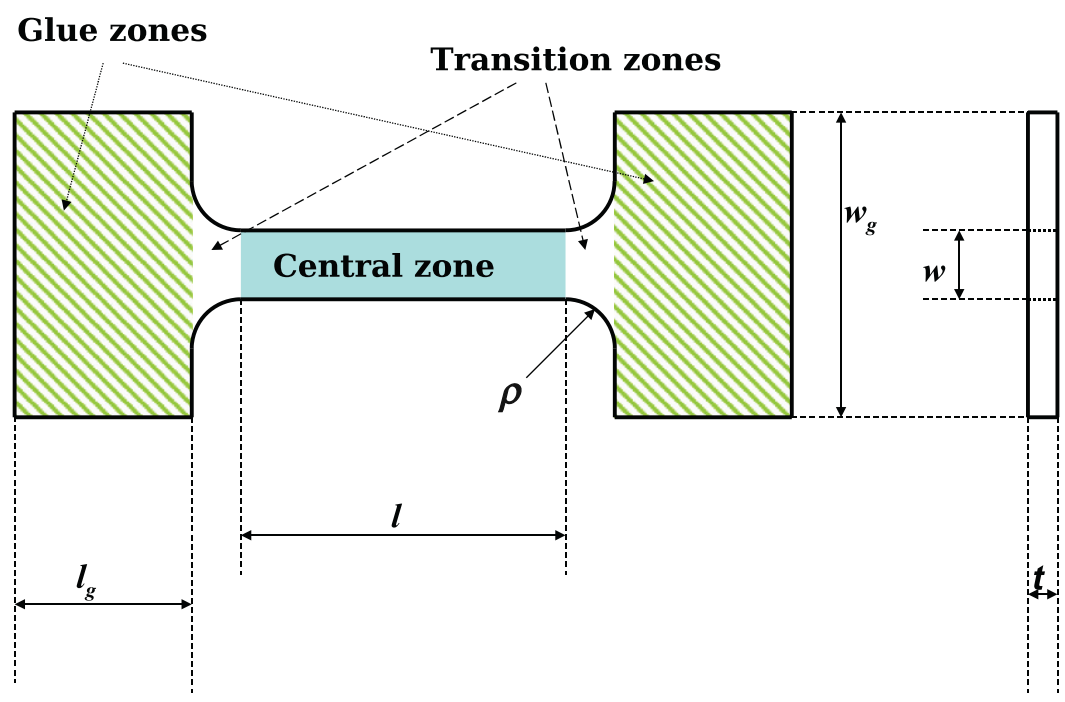

Fig. 1. Specimen geometry. 


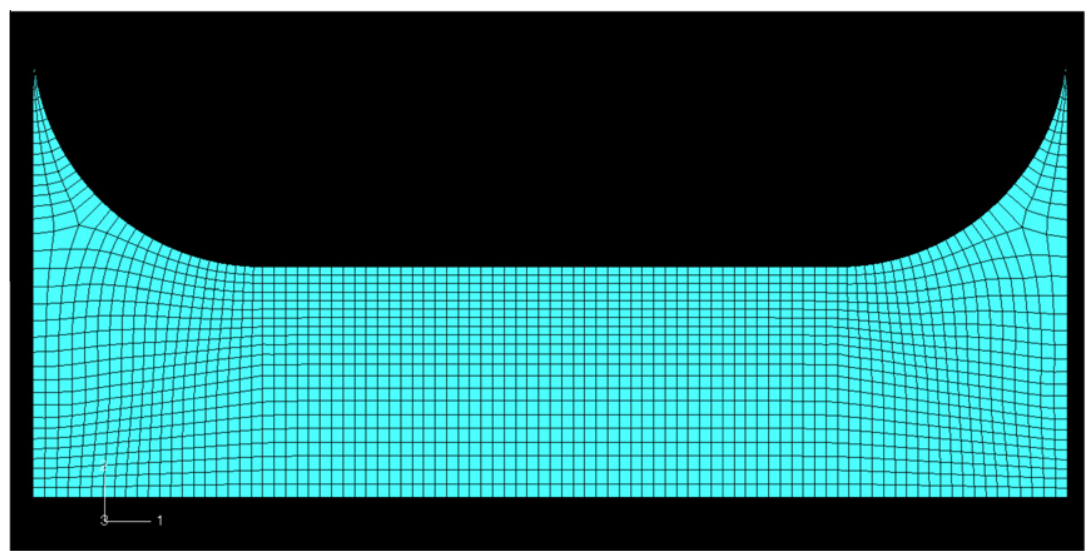

Fig. 2. Finite-element mesh.

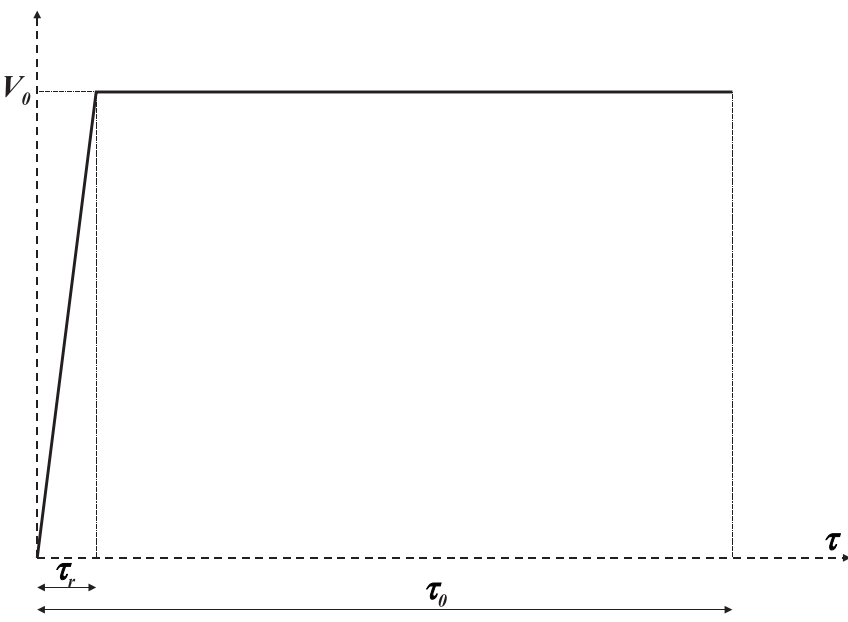

Fig. 3. Velocity profile.

length of the central zone and the two transitions zones, we obtain a second measurement of the nominal strain:

$\epsilon_{n}^{\prime}(\tau)=\frac{\mathcal{U}^{A b}(\tau)}{l+2 \rho}$

where $\rho$ is radius of the transition zones. Nevertheless, it is required, for Eq. (4), to suppose that the strain field is homogeneous in the whole central and transition zones. This assumption is only valid for low values of $\rho$.

The nominal stress and strain are computed by considering the cross-section area and sample length at the beginning of the test. We can also determine the true stress and strain by considering the evolution of the sample length and cross-sectional area. If we consider the first strain measure (Eq. (3)), the true (logarithmic) strain reads:

$\epsilon_{t}(\tau)=\log \left(1+\epsilon_{n}(\tau)\right)$

and with the second strain measure (Eq. (4)), the true (logarithmic) strain reads:

$\epsilon_{t}^{\prime}(\tau)=\log \left(1+\epsilon_{n}^{\prime}(\tau)\right)$.

On the other hand, the true stress can be expressed as:

$\sigma_{t}(\tau)=\frac{\sigma_{n}(\tau)}{\left(1-v \epsilon_{n}(\tau)\right)^{2}}$

where $v$ is the Poisson ratio.

\subsubsection{Error measurement}

We are interested in quantifying errors on stress and strain in dynamic tensile experiments. Therefore, we will compare the strain and stress obtained by Eqs. (5)-(7) to the strain and stress obtained directly by finite-element simulations.

The true strain measures given in Eqs. (5) and (6) are estimations of the average axial strain in central zone. With Abaqus, we can have the logarithmic strain field. We consider $\underline{\mathbf{X}}=x_{1} \underline{\mathbf{e}}_{1}+x_{2} \underline{\mathbf{e}}_{2}$ a point of the central zone. Let $\epsilon_{11}^{A b}\left(x_{1}, x_{2}, \tau\right)$ be the first diagonal component of strain field at this point. Then, the average strain in the central zone reads:

$$
\left\langle\epsilon_{11}^{A b}\right\rangle(\tau)=\frac{\int_{x_{1}} \int_{x_{2}} \epsilon_{11}^{A b}\left(x_{1}, x_{2}, \tau\right) d x_{2} d x_{1}}{\int_{x_{1}} \int_{x_{2}} d x_{2} d x_{1}} .
$$

The error on the first strain measure is defined by:

$\xi_{\epsilon}(\tau)=\frac{\left|\left\langle\epsilon_{11}^{A b}\right\rangle(\tau)-\epsilon_{t}(\tau)\right|}{\left\langle\epsilon_{11}^{A b}\right\rangle(\tau)}$.

Similarly, the error on the second strain measure is defined by:

$\xi_{\epsilon^{\prime}}(\tau)=\frac{\left|\left\langle\epsilon_{11}^{A b}\right\rangle(\tau)-\epsilon_{t}^{\prime}(\tau)\right|}{\left\langle\epsilon_{11}^{A b}\right\rangle(\tau)}$.

By finite-element simulations we have also the Cauchy stress field in central zone. Let $\sigma_{11}^{A b}\left(x_{1}, x_{2}, \tau\right)$ be the first diagonal component of strain field at the point $\underline{\mathbf{X}}$. The average stress in the central zone is expressed as:

$$
\left\langle\sigma_{11}^{A b}\right\rangle(\tau)=\frac{\int_{x_{1}} \int_{x_{2}} \sigma_{11}^{A b}\left(x_{1}, x_{2}, \tau\right) d x_{2} d x_{1}}{\int_{x_{1}} \int_{x_{2}} d x_{2} d x_{1}} .
$$

This average value should be compared to the stress measure in Eq. (7). However, this expression involves the nominal strain. In order to separate the problems on stress and strain measurements, we express the stress in terms of the average strain obtained by finite-element simulations:

$\sigma_{t}^{\prime}(\tau)=\frac{\sigma_{n}(\tau)}{\left(1-v \mathrm{e}^{\left\langle\epsilon_{11}^{A b}\right\rangle(\tau)}\right)^{2}}$.

Then, the error on the stress measure is defined by:

$\xi_{\sigma}(\tau)=\frac{\left|\left\langle\sigma_{11}^{A b}\right\rangle(\tau)-\sigma_{t}^{\prime}(\tau)\right|}{\left\langle\sigma_{11}^{A b}\right\rangle(\tau)}$

Above, we defined the strain and stress error coefficients: $\xi_{\epsilon}(\tau), \xi_{\epsilon^{\prime}}(\tau)$ and $\xi_{\sigma}(\tau)$. In order to understand the errors origin, we study also the correlation between these errors and the stress 
and strain homogeneity. Then, two homogeneity coefficients are defined to measure the strain and stress homogeneity during the test (simulation). The strain homogeneity coefficient reads:

$\alpha_{\epsilon}(\tau)=\frac{\int_{x_{1}} \int_{x_{2}}\left|\epsilon_{11}^{A b}\left(x_{1}, x_{2}, \tau\right)-\left\langle\epsilon_{11}^{A b}\right\rangle(\tau)\right| d x_{2} d x_{1}}{\int_{x_{1}} \int_{x_{2}}\left|\left\langle\epsilon_{11}^{A b}\right\rangle(\tau)\right| d x_{2} d x_{1}}$.

Similarly, the strain homogeneity coefficient reads:

$\alpha_{\sigma}(\tau)=\frac{\int_{x_{1}} \int_{x_{2}}\left|\sigma_{11}^{A b}\left(x_{1}, x_{2}, \tau\right)-\left\langle\sigma_{11}^{A b}\right\rangle(\tau)\right| d x_{2} d x_{1}}{\int_{x_{1}} \int_{x_{2}}\left|\left\langle\sigma_{11}^{A b}\right\rangle(\tau)\right| d x_{2} d x_{1}}$.

These coefficients measure the mean difference between the stress and strain fields and their average values. The stress and strain fields are called homogeneous when $\alpha_{\epsilon}$ and $\alpha_{\sigma}$, respectively, tends to 0 .

\section{Results}

\subsection{Reference model}

Before considering the influence of several parameters, we will first discuss results obtained with the reference model. In Fig. 4(a), we plot the stress as can be measured experimentally (Eq. (12)) and the reference stress obtained by finite-element simulations
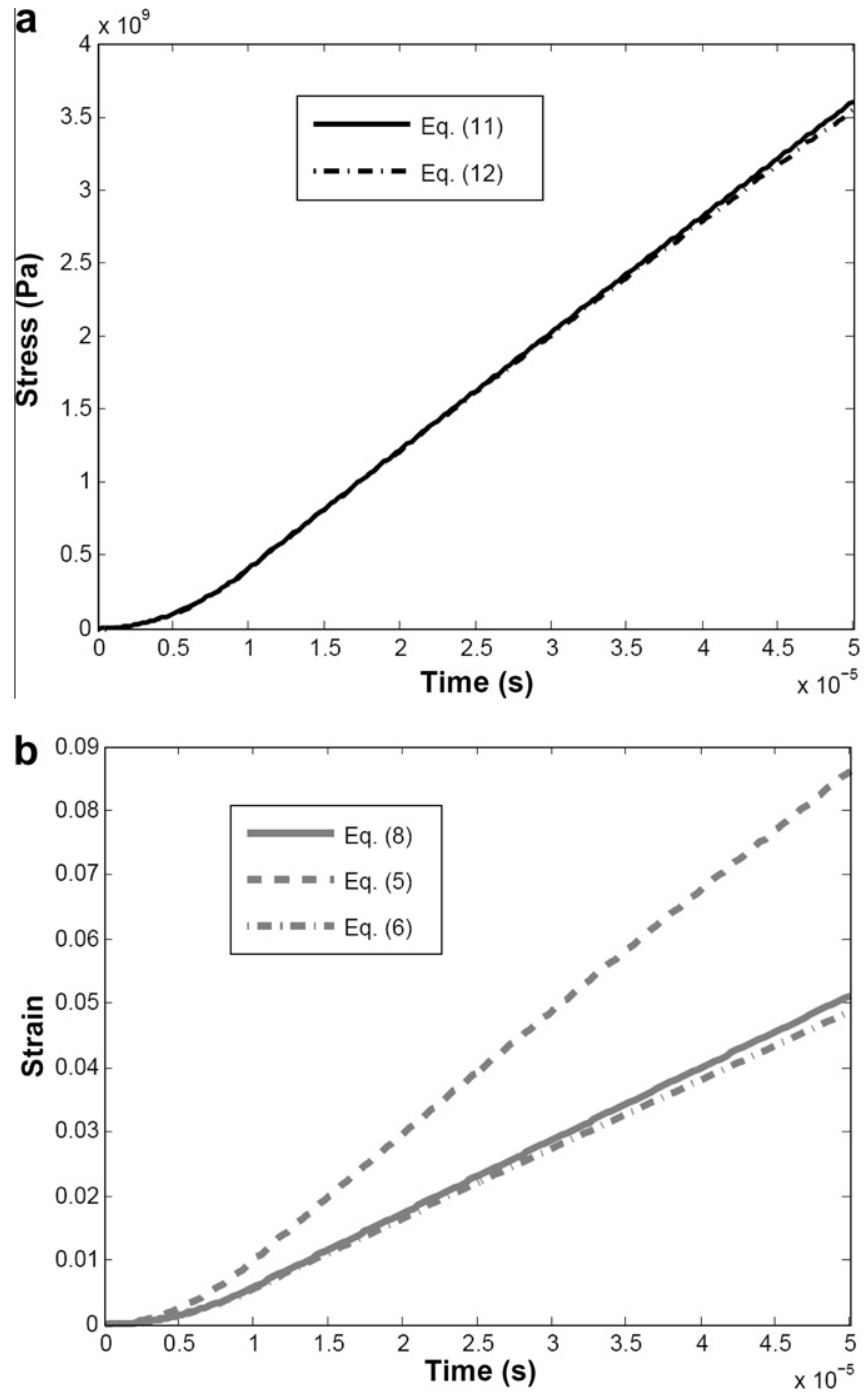

Fig. 4. Comparison between different stress (a) and strain (b) measures.
(Eq. (11)). It comes from this plot that the two stress curves are superposed and no major difference exists. Similarly, we plot, in Fig. 4(b), the reference strain obtained by finite-element simulations (Eq. (8)) and the two experimental-like strain measures defined in Eqs. (5) and (6). Contrarely to the experimental-like stress measure, the strain measures show some differences with the reference strain. Note that when considering only the central zone length (Eq. (5)), the measured strain is significantly different from the reference strain. Moreover, this first strain measure overestimates the strain because it assumed that only the central zone is deformed. However, the second strain measure (Eq. (6)) underestimates the strain as it assumes that the central and transition zones are deformed uniformly.

Furthermore, we plot, in Fig. 5(a), the errors on the strain and stress measures as defined in Eqs. (9), (10) and (13). In the beginning of the test (simulation), the errors are important for the three measures (one stress and two strain). This is during few microseconds. The errors are higher than 0.1 (10\%). Most likely, the beginning of the test is governed by the wave propagation effects. Indeed, parts of the specimen are loaded and others not during the first micro-seconds. Hence, the stress and strain fields are highly heterogeneous. After this transient behaviour, the error on the stress seems to vanish. It is lower than 0.02 (2\%). The error at the end of simulation is $1.53 \%$. Consequently, the assumption, that
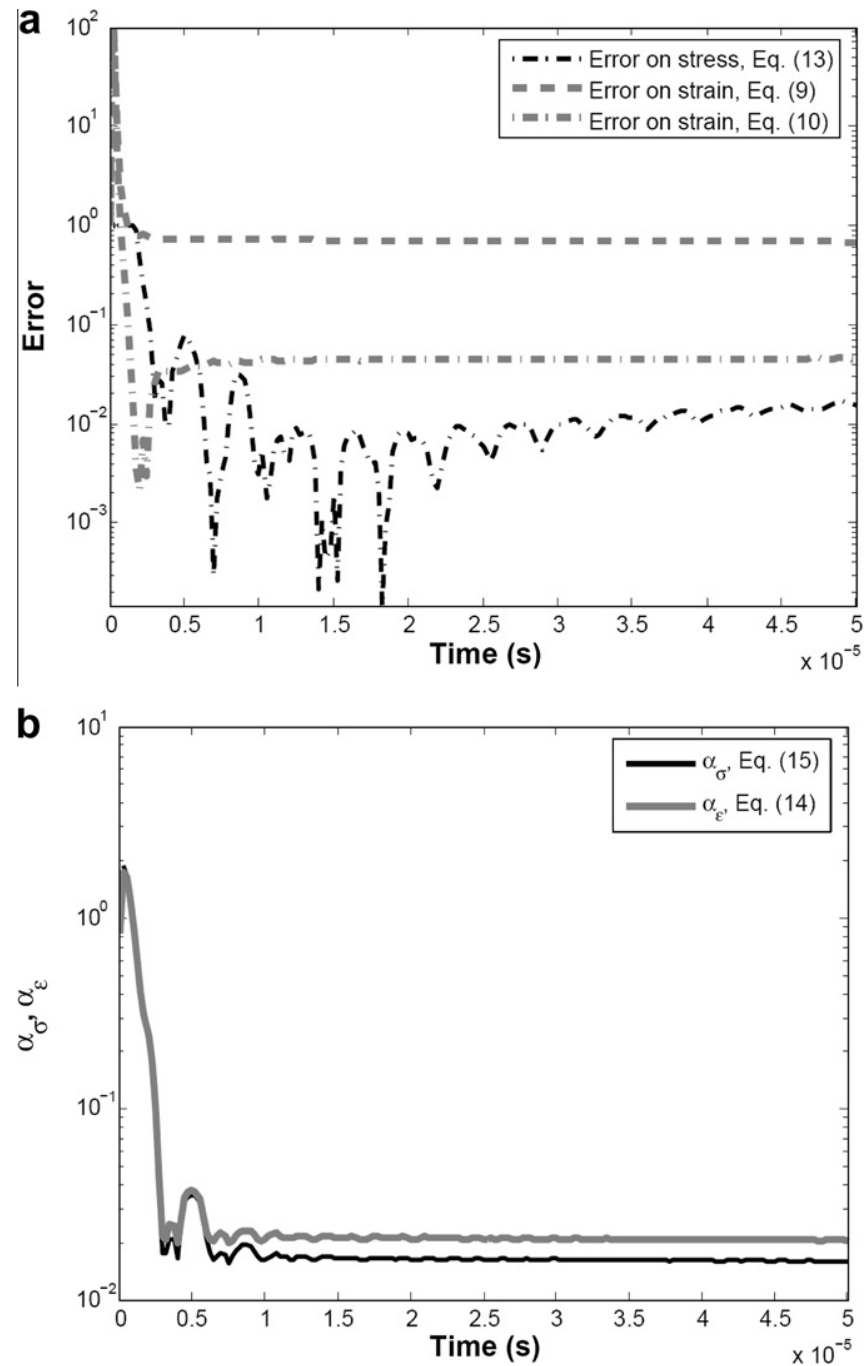

Fig. 5. (a) Errors on the stress and strain measures, and (b) stress and strain homogeneity coefficients. 
the force applied at any cross-section of the specimen is the same, is valid. On the other hand, the error of the first strain measure, which takes into account only the central zone length, is important. It is always higher than $65 \%$. This means that not only the central zone is deformed but also the transition zones. The second strain measure gives better approximation. Except the beginning of the test, the error is about $4.5 \%$. The major problem of the second strain measure is that it assumes the transition zones are deformed is the same way as the central zone, which cannot be true. However, the second strain measure seems to be valid at least for the reference model.

Now, we evaluate also the stress and strain homogeneity. In Fig. 5(b), we plot strain and stress homogeneity coefficients, defined in Eqs. (14) and (15), respectively. The plot reveals that the two coefficients have the same tendency. They have important values in the beginning of the test then they vanish. The stress and strain fields are heterogeneous during few micro-seconds and homogeneous thereafter. Generally, it is assumed that stress or strain fields are heterogeneous if the homogeneity coefficient is higher than $5 \%$. In the case of the reference model, we find that the stress and strain fields are homogeneous for $\tau \geqslant \tau_{h}=2.70 \mu \mathrm{s}$. Let $C_{0}$ be the sound speed in the specimen material considered in the reference model. In the case of the aluminum-like material used in the reference model $C_{0}=5000 \mathrm{~m} / \mathrm{s}$. During, a time $\tau_{h}$, waves propagate at a velocity $C_{0}$ through a distance $\delta$ expressed as:

$\delta=C_{0} \times \tau_{h}$.

In the case of the reference model, $\delta=13.5 \mathrm{~mm}$. This distance $\delta$ is approximately $2.7 \times l$ and $1.5 \times(l+2 \rho)$. This means that the tensile wave in the specimen, should make 2.7 times the central zone length or 1.5 times the length of the whole central and two transition zones, in order to achieve the stress and strain homogeneity. As stated above, the stress and strain heterogeneity may explain the errors on stress and strain measures in the beginning of the test. Since $\alpha_{\epsilon}$ has the same tendency as $\alpha_{\sigma}$, we limit our study to the second coefficient.

\subsection{Influence of the material}

In the reference model, we consider an aluminum-like material. In order to determine the influence of the material, we study four other materials. All the other testing and geometrical parameters are kept unchanged. Details about materials are given in Table 1.

$\xi_{\epsilon}, \xi_{\epsilon^{\prime}}, \xi_{\sigma}$ and $\alpha_{\sigma}$ are shown in Fig. 6(a)-(d). Note that we plot these parameters for times lower than $5 \mu$ s as almost no change is observed after this time. Indeed, the material influences only the errors in the beginning of the test, i.e., the transitional regime. It has almost no influence on the permanent part. An explanation to this would be the sound speed. The higher the sound speed is, the faster homogeneity is achieved.

Fig. 6(a)-(c) depict the evolution of the error on the strain and stress measures. Once the peak is reached, the errors decrease in the same way for aluminum and steel. Indeed, the sound speed in aluminum and steel is almost the same. The errors decrease less rapidly for cast-iron, brass and PMMA than for steel and aluminum. Comparing cast iron, brass and PMMA, the errors decrease faster in

Table 1

Characteristics of used materials.

\begin{tabular}{llll}
\hline Material & Young's modulus (MPa) & Poisson ratio & Density $\left(\mathrm{kg} / \mathrm{m}^{3}\right)$ \\
\hline Aluminum-like & 70 & 0.34 & 2800 \\
Steel-like & 200 & 0.3 & 7800 \\
Cast iron-like & 150 & 0.29 & 7800 \\
Brass-like & 110 & 0.35 & 8500 \\
PMMA-like & 10 & 0.4 & 1200 \\
\hline
\end{tabular}

the case of the first material, than the second and than the third. This can be explained by the sound speed which is higher in cast iron than it is in brass and than in PMMA.

The same tendency is observed in Fig. 6(d). The decrease of $\alpha_{\sigma}$ is the fastest in the case of aluminum and steel and is lowest in the case of PMMA. The stress and strain homogeneities can be explained by wave propagation. For materials with high sound speed, the homogeneity is reached faster than for the other cases. It seems also that the errors on stress and strain measures and stress tri-axiality are linked to the stress and strain homogeneities.

In order to understand the influence of the material on the homgenisation process, we plot the time $\tau_{h}$ in terms of the material sound speed $C_{0}$ (Fig. 7). It comes out that $\tau_{h}$ is inversely proportional to $C_{0}$. This assertion is verified when computing the distance $\delta=C_{0} \times \tau_{h}$ defined in Eq. (16). This distance is equal to $13.6^{ \pm 0.1} \mathrm{~mm}$ for the five considered materials. Therefore, the loading wave should move through the same distance $\delta$ in order to achieve stress and strain heterogeneity. This distance $\delta$ does not depend on the material but it should depend on the specimen geometry or the loading rise time. This will be investigated later.

\subsection{Influence of the specimen geometry}

In order to determine the optimum sample geometry we investigate the influence of several parameters on the errors on stress and strain measures. Precisely, we investigate the length and width of the central zone and the radius of the transition zones.

\subsubsection{Central zone Length}

In this section we investigate the influence of the central zone length on the stress and strain measures errors. We considered six values of the length: 1, 3, 5, 10, 20 and $50 \mathrm{~mm}$. The 5-mm long central zone is the case of the reference model. We plot $\xi_{\epsilon}, \xi_{\epsilon^{\prime}}, \xi_{\sigma}$ and $\alpha_{\sigma}$ in Fig. 8(a)-(d), respectively, for the six values of the central zone length. Moreover, we synthesize in Fig. 9(a) the influence of this length.

The error $\xi_{\epsilon}$, of the first strain measure $\epsilon_{t}$, significantly decreases with the central zone length (Fig. 8(a)). This is quite unsurprising. Indeed, the transition zones length tends to be insignificant compared to the central zone length when this last increases. Therefore, the imposed displacement $\mathcal{U}^{A b}(\tau)$ is mostly applied to the central zone for high values of central zone length $l$. In this last case, the displacements of the transition zones can be neglected. Hence, Eq. (3), and consequently Eq. (5), are valid. Excepting the beginning of the test, the error is almost constant, independently of the central zone length. Therefore, we plot, in Fig. 9, the error by the end of the simulation (when the average logarithmic strain equals $5 \%$ ). It is worth to notice that this error is higher than $100 \%$ for $l$ lower than $3 \mathrm{~mm}$. It slows down to only $6.7 \%$ when $l=50 \mathrm{~mm}$.

Similarly to $\xi_{\epsilon}$, the error $\xi_{\epsilon^{\prime}}$, of the first strain measure $\epsilon_{t}^{\prime}$, decreases with the central zone length (Fig. 8(b)). This is less foreseeable. Indeed, the second strain measure is introduced to correct the effects of the transition zones. However, increasing the central zone length leads to diminish the transition zones effect. Nevertheless, the fact that the error drops with length $l$ may be explained by strain field homogeneity. Indeed, the second strain measure (Eqs. (4) and (6)), assumes that the strain field is uniform in the whole central and transition zones. This means that the central zone deforms in the same way as the transition zones. But, this is not true. After the transient regime $\left(\tau>\tau_{h}\right)$, the strain field is almost homogeneous in central zone. Increasing the length $l$ yield a significant weight to central zone strain field compared to the transition zones strain field. The strain field in whole central and transition zones will be more homogeneous with important values of the central zone length. That is why the error on the second measure decreases when this length rises. The error on the second measure 

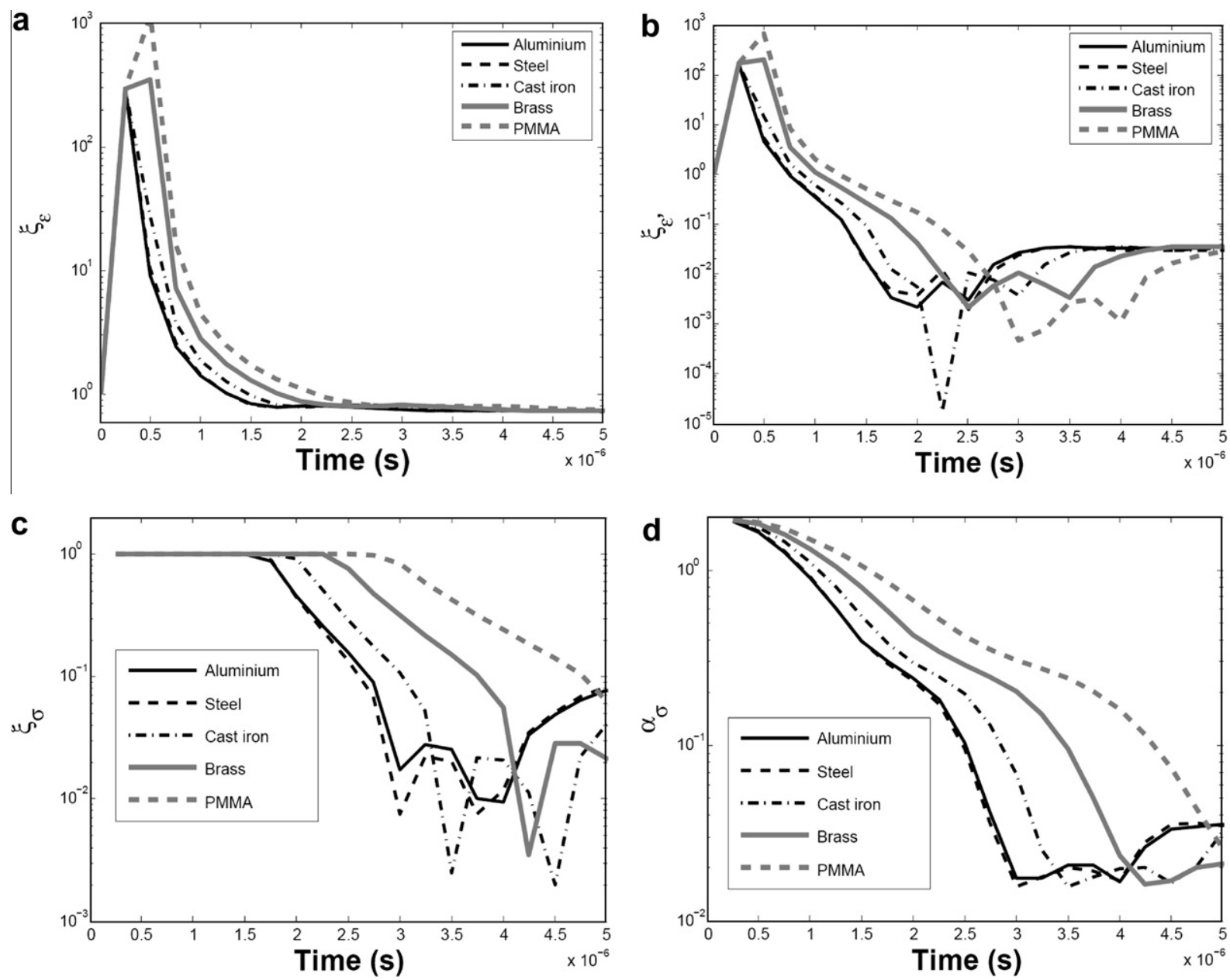

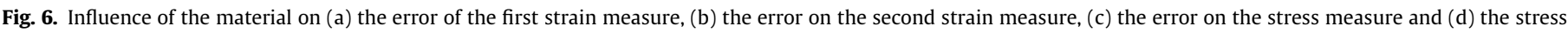
homogeneity coefficient.

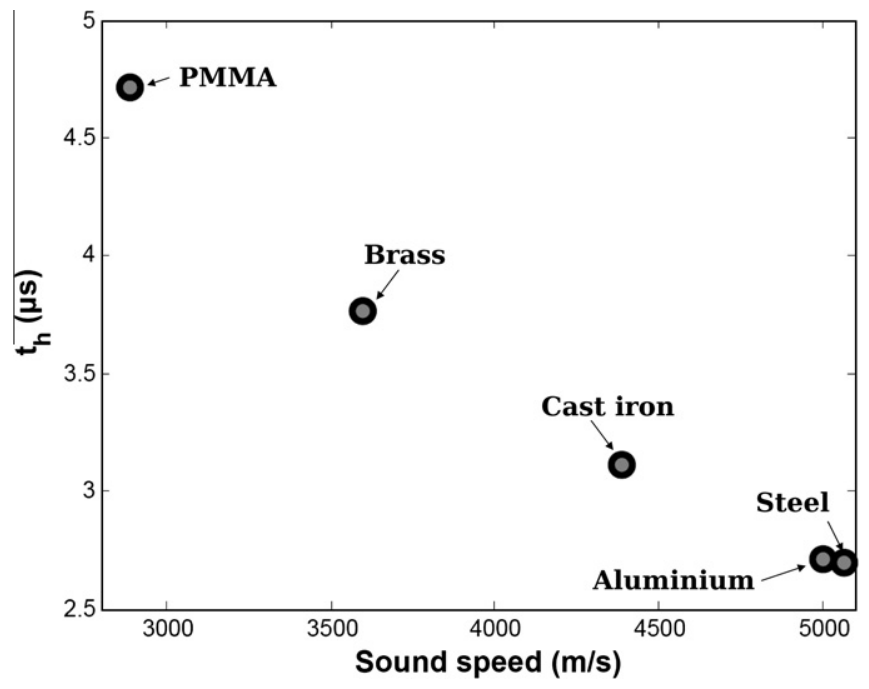

Fig. 7. The time $\tau_{h}$ needed by the stress and strain fields to homogenise.

is also constant excepting the beginning of test. This constant value is plotted in terms of the length $l$ in Fig. 9. It is equal to $8.2 \%$ and $6.1 \%$ for $l=1 \mathrm{~mm}$ and $l=3 \mathrm{~mm}$. It is lower than $5 \%$ for $l$ lower than $5 \mathrm{~mm}$. Independently of the length $l$ the second strain measure gives better results than the first.
We plot the error $\xi_{\sigma}$ on the stress measure $\sigma^{\prime}$ in Fig. 8(c). For length $l$ lower than $20 \mathrm{~mm}$, the error drops sharply in the beginning of the simulation. Subsequently, it oscillates around quite constant value. For the longest sample, $l=50 \mathrm{~mm}$, the error drops slowly when oscillating. Furthermore, we plot the time average of $\xi_{\sigma}\left[\xi_{\sigma}\right]$, in Fig. 9. The average error is about $2.2 \%$ for $l=1 \mathrm{~mm}$. It drops to $1.6 \%$ when $l=5 \mathrm{~mm}$ and rises thereafter to reach $6.5 \%$ for $l=50 \mathrm{~mm}$.

The error on the stress measure can be explained by the stress field homogeneity. In Fig. 8(d), we show the time variation of the stress homogeneity coefficient for the different values of the length $l$. We remark that, for $l=1,3,5$ and $10 \mathrm{~mm}$, the homogeneity coefficients drop rapidly in the beginning of the test. It takes a constant value thereafter. However, this coefficient slowly decreases while oscillating for $l=20$ and $50 \mathrm{~mm}$. This can be interpreted by the origin of the stress heterogeneity. For low central zone lengths, there are two origins of the stress field heterogeneity: geometrical and dynamic. Precisely, the sample geometry creates stress concentration regions near the transition-central zones frontiers. This induces geometrical stress field heterogeneity and can be observed even in quasi-static conditions. This heterogeneity should be independent of time. Besides, due to the finite velocity of waves, any load needs short time to be transmitted to whole of the sample. This is the dynamic stress field heterogeneity which can be neglected for quasi-static tests. The shorter the specimen is, the faster this heterogeneity disappears. Furthermore, this heterogeneity has an oscillating behaviour. Its characteristics period corresponds to 

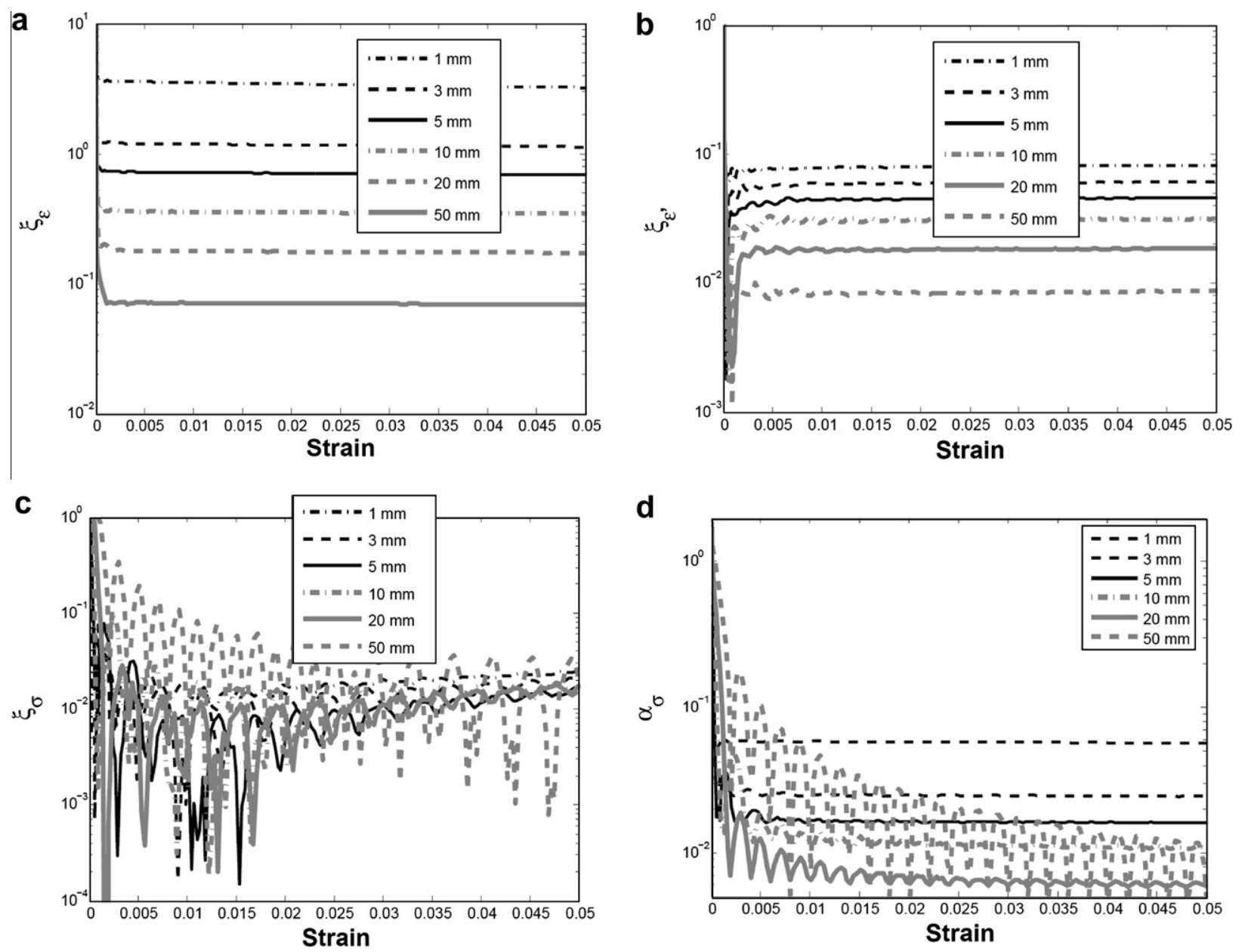

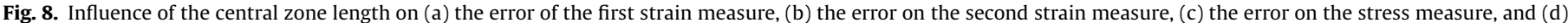
the stress homogeneity coefficient.

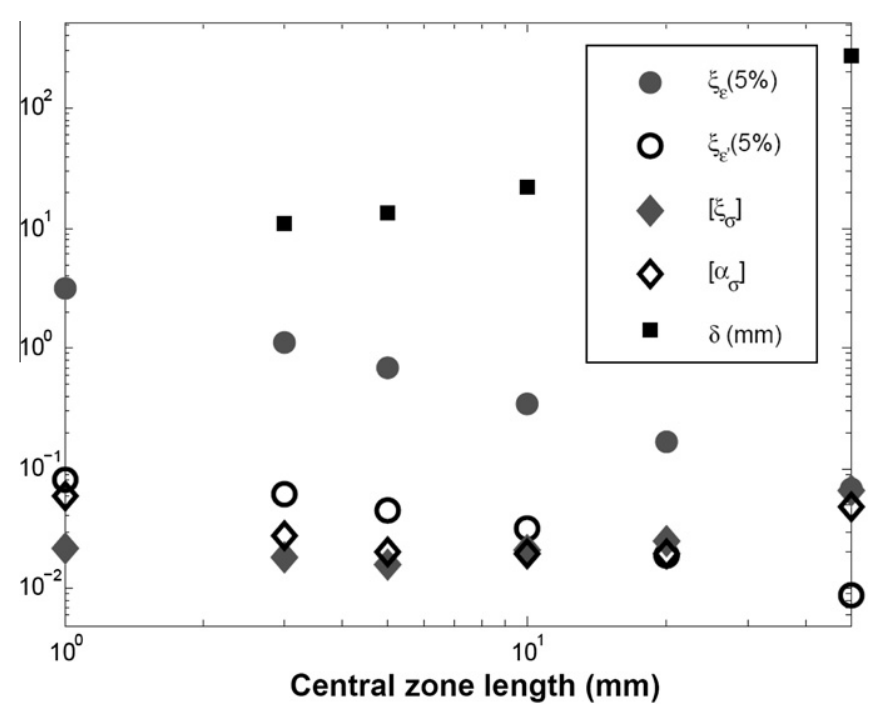

Fig. 9. Influence of the central zone length on several parameters.

the time needed by the wave to travel a characteristics distance. In the case of low values of $l$, the dynamic heterogeneity vanishes rapidly. After few micro-seconds, it disappears and only the geometrical heterogeneity remains. For important values of the length $l$, the geometrical heterogeneity is insignificant. Moreover, the dynamic heterogeneity vanishes slowly. The time average of the homogeneity coefficient $\left[\alpha_{\sigma}\right]$, is shown in Fig. 9. It is decreasing for $l$ lower than $5 \mathrm{~mm}$. Then, it has an almost constant value up to $20 \mathrm{~mm}$ and increases for $l$ higher than $20 \mathrm{~mm}$. It is clear from this analysis, that the error on the stress measure and stress homogeneity coefficient have the same tendency. It can be concluded that the stress field heterogeneity is the main cause of errors on the stress measure.

\subsubsection{Transition zones radius}

In order to investigate the influence of the transitions zones radius, seven values of this radius are considered: $0.5,1,2,3,4,5$ and $10 \mathrm{~mm}$. The radius $2 \mathrm{~mm}$ corresponds to the reference model. In Fig. 10(a) and (b), we plot the errors on the first and second strain measures, respectively. These two errors have a sharp drop in the beginning of the test and they stabilize thereafter. The sharp drop corresponds to the dynamic homogenisation process. Contrarily to results of the previous section, the increase of the radius $\rho$ tends to rise the errors on strain measurements. This is for the same reasons explained at Section 3.3.1. Precisely, the increase of the radius $\rho$ make the contribution of the transition zones significant in the deformation process, which is neglected in Eqs. (3) and (5). That is why the error $\xi_{\epsilon}$ increases with the transition zones radius (see also Fig. 11). From this last figure, the lowest constant value of $\xi_{\epsilon}$ is $17.8 \%$ obtained with the lowest radius $\rho=0.5 \mathrm{~mm}$. On the other hand, the strain field is homogeneous in the central zone after few micro-seconds, which is not the case in the transition 

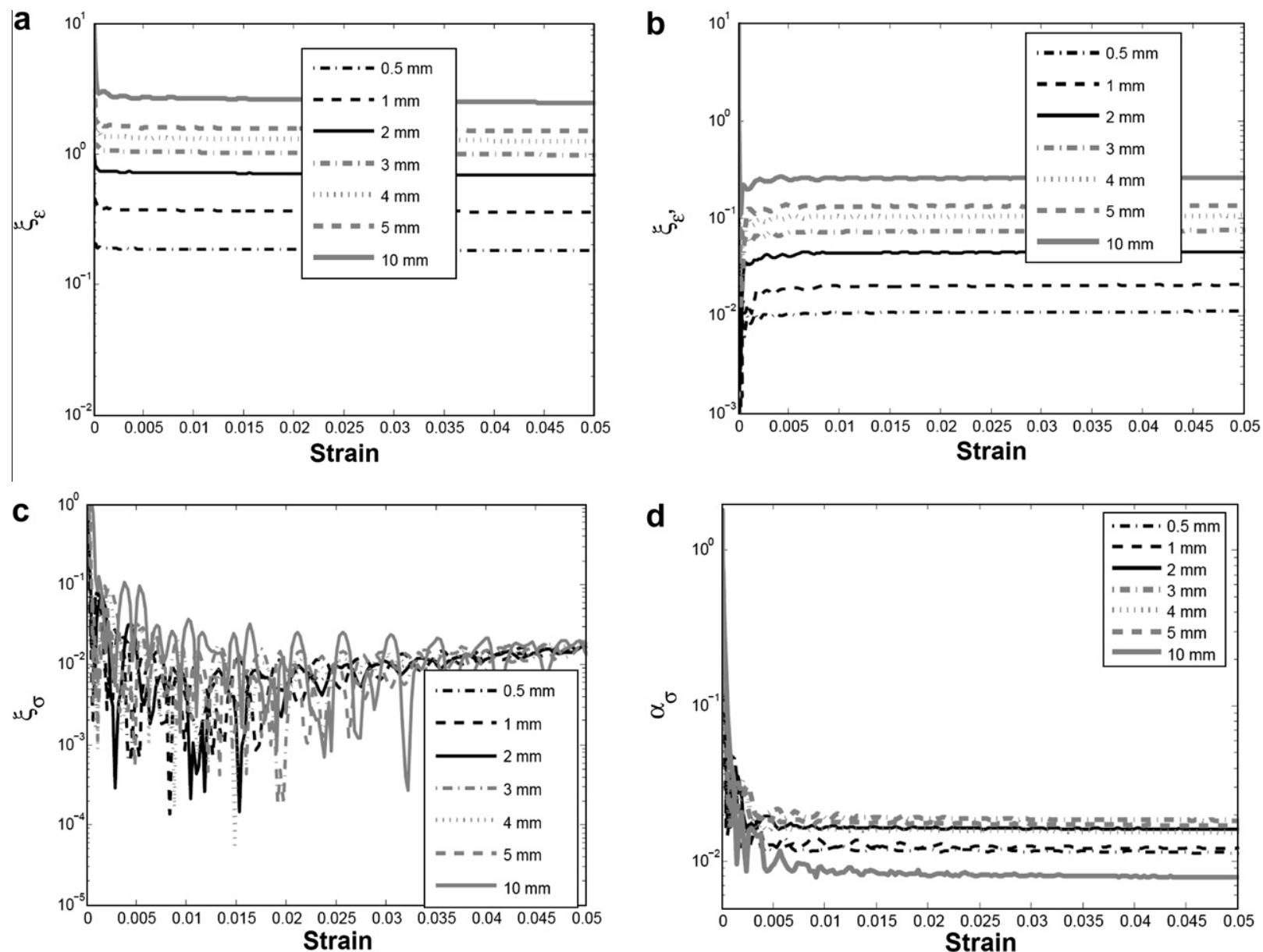

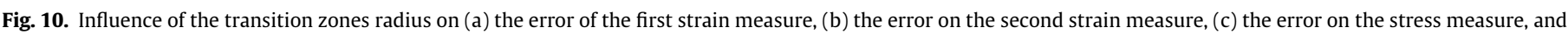
(d) the stress homogeneity coefficient.

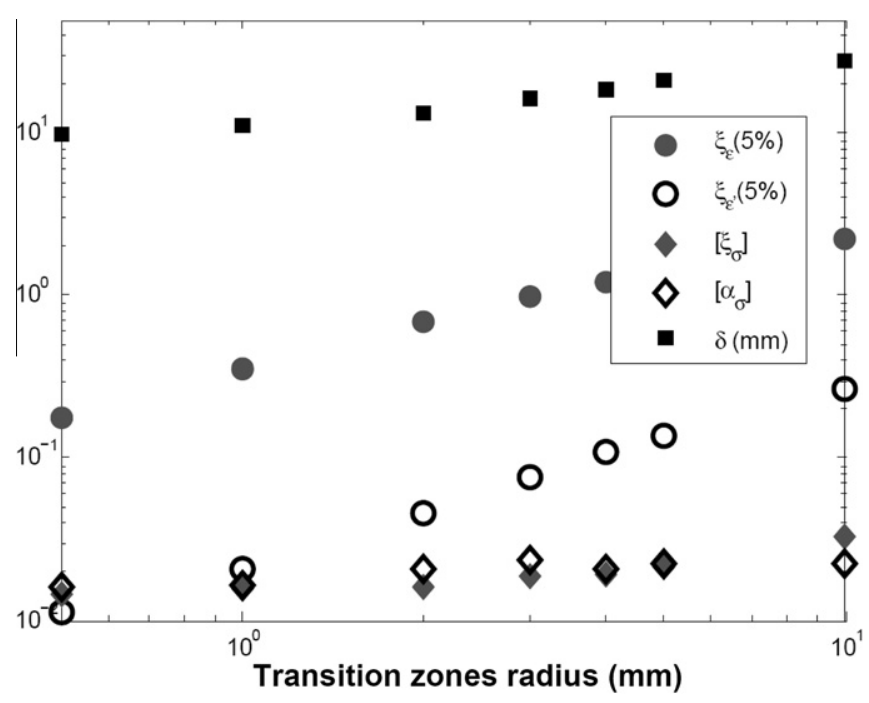

Fig. 11. Influence of the transition zones radius on several parameters.

zones. Hence, the increase of the radius $\rho$ makes stress field more heterogeneous in the whole central and transition zones. That is why the error $\xi_{\epsilon^{\prime}}$ also increases with the transition zones radius (see Fig. 11). It is worth to notice that for all considered radii, $\xi_{\epsilon^{\prime}}$ is always lower than $\xi_{\epsilon}$. Moreover, the constant value of $\xi_{\epsilon^{\prime}}$ is lower than $10 \%$ for $\rho<4 \mathrm{~mm}$.
Fig. 10(c) and (d) show the error on the stress measure and the stress homogeneity coefficient, respectively. Both parameters rise with the radius $\rho$ (see also Fig. 11). Indeed, when the radius increases, the loading wave has to do longer distance and the stress homogenisation process takes more time. This remark is verified in Fig. 11 which depicts that the distance $\delta$ increases $\rho$. This distance is equal to $9.9 \mathrm{~mm}$ for the lowest radius and grow up to $27.95 \mathrm{~mm}$ for $\rho=10 \mathrm{~mm}$. We should notice that the increase in $\xi_{\sigma}$ and $\alpha_{\sigma}$ with the radius $\rho$ is smooth. The average value of $\xi_{\sigma}$ increases from $1.5 \%$ for $\rho=0.5 \mathrm{~mm}$ to achieve $3.3 \%$ for the highest radius, i.e., $\rho=10 \mathrm{~mm}$. On the other hand, the average value of $\alpha_{\sigma}$ rises to $1.6 \%$ for lowest radius to reach $2.3 \%$ for the highest radius.

\subsubsection{Influence of the central zone width}

The third geometrical parameter, investigated in this paper, is the central zones width. Six values are considered: $0.5,1,2,4,7$ and $10 \mathrm{~mm}$. The case of 4 -mm-width geometry corresponds to the reference model.

We plot in Fig. 12(a), the error on the first strain measure. After a sharp drop, due to dynamic strain field homogenisation, the error takes an almost constant value which increases with the width $w$ (Fig. 13). It is equal to $44.3 \%$ for $w=0.5 \mathrm{~mm}$ and grow up to $70.1 \%$ for the largest geometry $(w=10 \mathrm{~mm})$. For the highest values of the width, this parameter is significantly higher than the transition zones radius. Therefore, the cross-sectional areas of the transition zone become of the same order than the central zone cross-section area. Hence, the strain in the transition zones is also of the same order as the strain in the central zone. Consequently, 

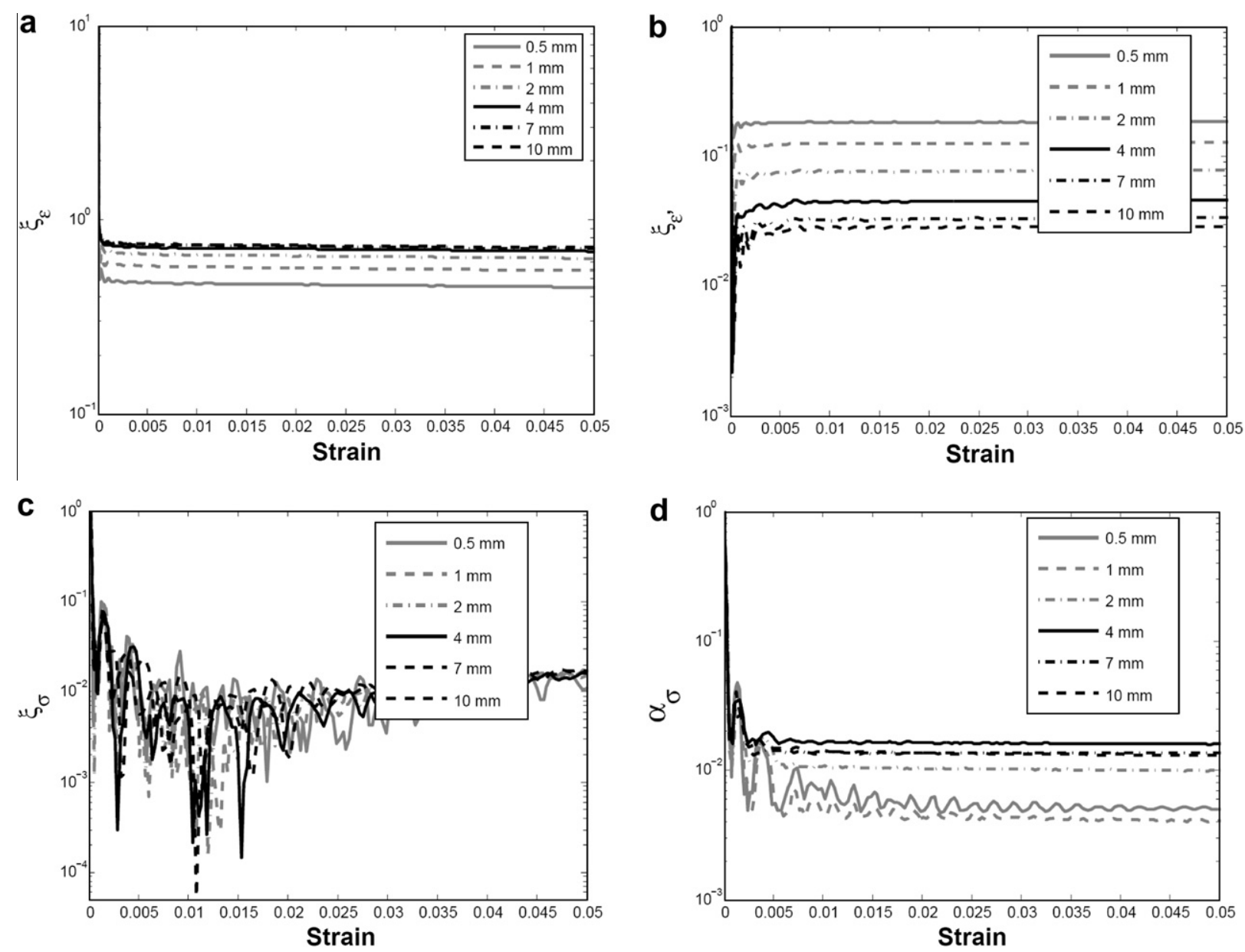

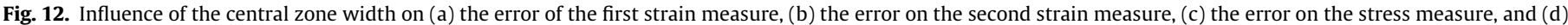
the stress homogeneity coefficient.

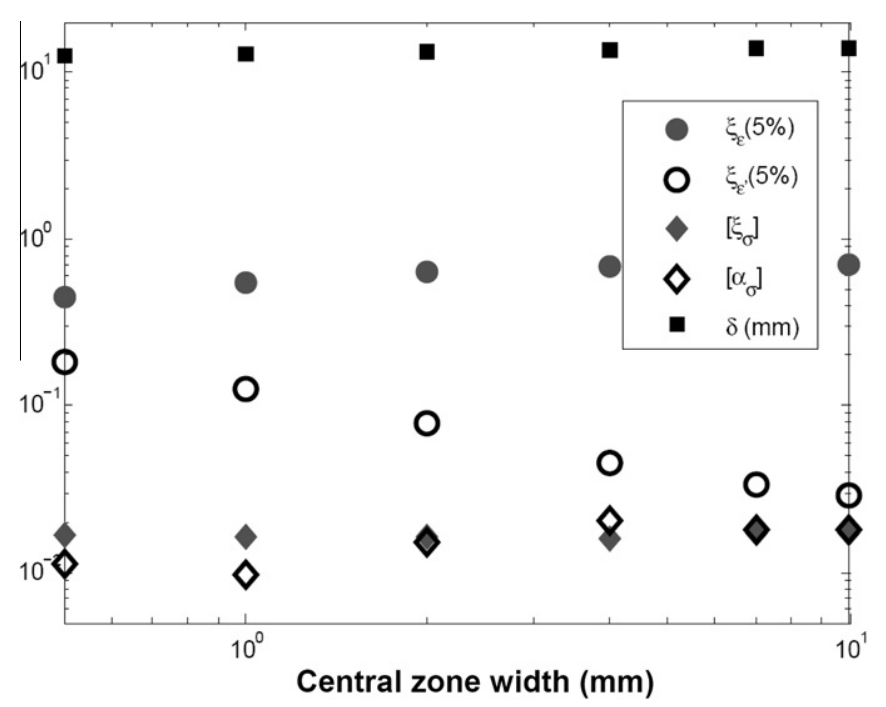

Fig. 13. Influence of the central zone width on several parameters.

the error of the first strain measure, which neglects the deformation of the transition zones, rises when increasing the width $w$. For the same reason the error of the second strain measure, which assumes that the deformation of the transition zones is of the same order as the deformation in the central zone, decreases when the width $w$ rises (Fig. 12(b) and Fig. 13). Excepting the first micro-second of the simulation (test), $\xi_{\epsilon^{\prime}}$ takes also a constant value which slow down with the width. The constant value equals $18.3 \%$ for the lowest width and goes down to reach $2.9 \%$ for the largest central zone.

Fig. 12(c) illustrates the time variation of the error on the stress measure for the six values of the width $w . \xi_{\sigma}$ is almost insensitive to the width $w$. The average value $\left[\xi_{\sigma}\right]$ is also plotted in Fig. 13. It is almost constant with the width $w$. It varies between $1.59 \%$ and $1.72 \%$.

Fig. 12(d) shows the time variation of the stress homogeneity coefficients for the different values of $w$. The dynamic heterogeneity, present in the first part of the test, disappears almost at the time. This gives a quasi-constant characteristic distance $\delta$ (Fig. 13). Nevertheless, the geometrical induced heterogeneity is slightly different. This gives an average value $\left[\alpha_{\sigma}\right]$ which vary slightly with width $w$ (Fig. 13). No clear tendency can be observed. $\left[\alpha_{\sigma}\right]$ is equal $1.1 \%$ for the lowest width. Then it goes down to reach $0.98 \%$ for $w=1 \mathrm{~mm}$. Subsequently, it increases to reach $2.1 \%$ when the width is equal to $4 \mathrm{~mm}$. Then, this value equals $1.8 \%$ for the largest central zone. The correlation between the error on the stress measure and the stress heterogeneity coefficient is less evident in the case of the central zone width. This is because the lowest values of both parameters. That is why we compute the correlation coefficient defined as:

$\chi\left(\left[\xi_{\sigma}\right],\left[\alpha_{\sigma}\right]\right)=\frac{\left[\xi_{\sigma}\right] \cdot\left[\alpha_{\sigma}\right]}{\left\|\left[\xi_{\sigma}\right]\right\|\left\|\left[\alpha_{\sigma}\right]\right\|}$, 
where holds for the Eucledian scalar product and \| $\|$ the Eucledian norm. We obtain: $r\left(\left[\xi_{\sigma}\right],\left[\alpha_{\sigma}\right]\right)=0.97$, which means that both parameters are highly correlated.

\subsection{Influence of testing parameters}

In this paper, we are also interested in studying the influence of two testing parameters in addition to the influence of the material and geometrical parameters. Precisely, we investigate the influence of the loading rise time and velocity.

\subsubsection{Influence of the rise time $\tau_{r}$}

In real dynamic experiments, it is very hard to impose a constant velocity from the beginning of the test. The time needed to achieve a constant loading velocity is called here rise time. We considered seven values of this parameter: $0.1,0.5,1,5,10,25$ and $50 \mu \mathrm{s}$.

We plot in Fig. 14(a) and (b), the time variation of errors on the first and the second strain measures. Since the influence of the $\tau_{r}$ is concentrated in the beginning of the test, only the first $15 \mu$ s are shown. Indeed, the rise time $\tau_{r}$ only influences the dynamic homogenisation process. We observe no influence for the permanent part of the simulation (see also Fig. 15). We should notice here that authors compute the time average of $\xi_{\epsilon}$ and $\xi_{\epsilon^{\prime}}$.

The error on the stress measure is shown in Fig. 14(c). Two kinds of behaviour are observed. The first behaviour is observed for $\tau_{r}$ higher than $5 \mu$ s. There is a sharp drop in the beginning of the simulation followed by an oscillating behaviour. The second behaviour is observed for $\tau_{r}$ lower than $1 \mu$ s. The drop is rather smooth and oscillating. The same conclusions can be made for the stress homogeneity coefficient shown in Fig. 14(d). A first behaviour is observed for $\tau_{r}$ higher than $5 \mu$ s and a second one is observed for $\tau_{r}$ lower than $1 \mu \mathrm{s}$. The time average values, $[\xi \sigma]$ and $\left[\alpha_{\sigma}\right]$, are plotted in Fig. 15. Both parameters decrease with the rise time. The slope is smooth for $\tau_{r}$ higher than $5 \mu$ s and lower than $1 \mu \mathrm{s}$. However, there is sharp drop between 1 and $5 \mu \mathrm{s}$. The transition is when the rise time $\tau_{r}$ is equal to the time needed to achieve homogeneity, i.e., $\tau_{h}$. It is worth to notice that $\tau_{h}$ depends on $\tau_{r}$. We plot in Fig. 15, the variation of the ratio

$r_{\tau}=\frac{\tau_{h}}{\tau_{h}(\infty)} \approx \frac{\tau_{h}}{\tau_{h}\left(\tau_{r}=50 \mu s\right)}$,

which is defined as the ratio of time needed for homogenisation $\tau_{h}$, divided by the same time needed for homogenisation when the rise time is infinite $\left(\tau_{h}(\infty)=\tau\left(\tau_{r}=\infty\right)\right)$. The time $\tau_{h}(\infty)$ is approximated by $\tau_{h}\left(\tau_{r}=50 \mu \mathrm{s}\right) \approx 2.71 \mu \mathrm{s}$. For $\tau_{r}$ lower than $1 \mu \mathrm{s}$, the ratio $r_{\tau}$ is higher than 3.15 , which means that $\tau_{h}$ is $8.5 \mu \mathrm{s}$. This means that $\tau_{r}>\tau_{h}$. On the other hand, for $\tau_{r}$ higher than $5 \mu \mathrm{s} r_{\tau} \approx 1$. Thus $\tau_{h} \approx 2.71 \mu \mathrm{s}$. In this case $\tau_{r}<\tau_{h}$. Therefore, we say that when $\tau_{r}<\tau_{h}$ we will get the first kind of behaviour and when $\tau_{r}>\tau_{h}$ we will get the second kind of behaviour. The characteristic distance $\delta$ which has the same tendency as $\left[\xi_{\sigma}\right]$ and $\left[\alpha_{\sigma}\right]$ is also plotted in Fig. 15.

\subsubsection{Influence of the velocity}

In this paper, we are interested also in the influence of the loading velocity. We considered six values: $0.5,1,5,10,20$ and $50 \mathrm{~m} / \mathrm{s}$.
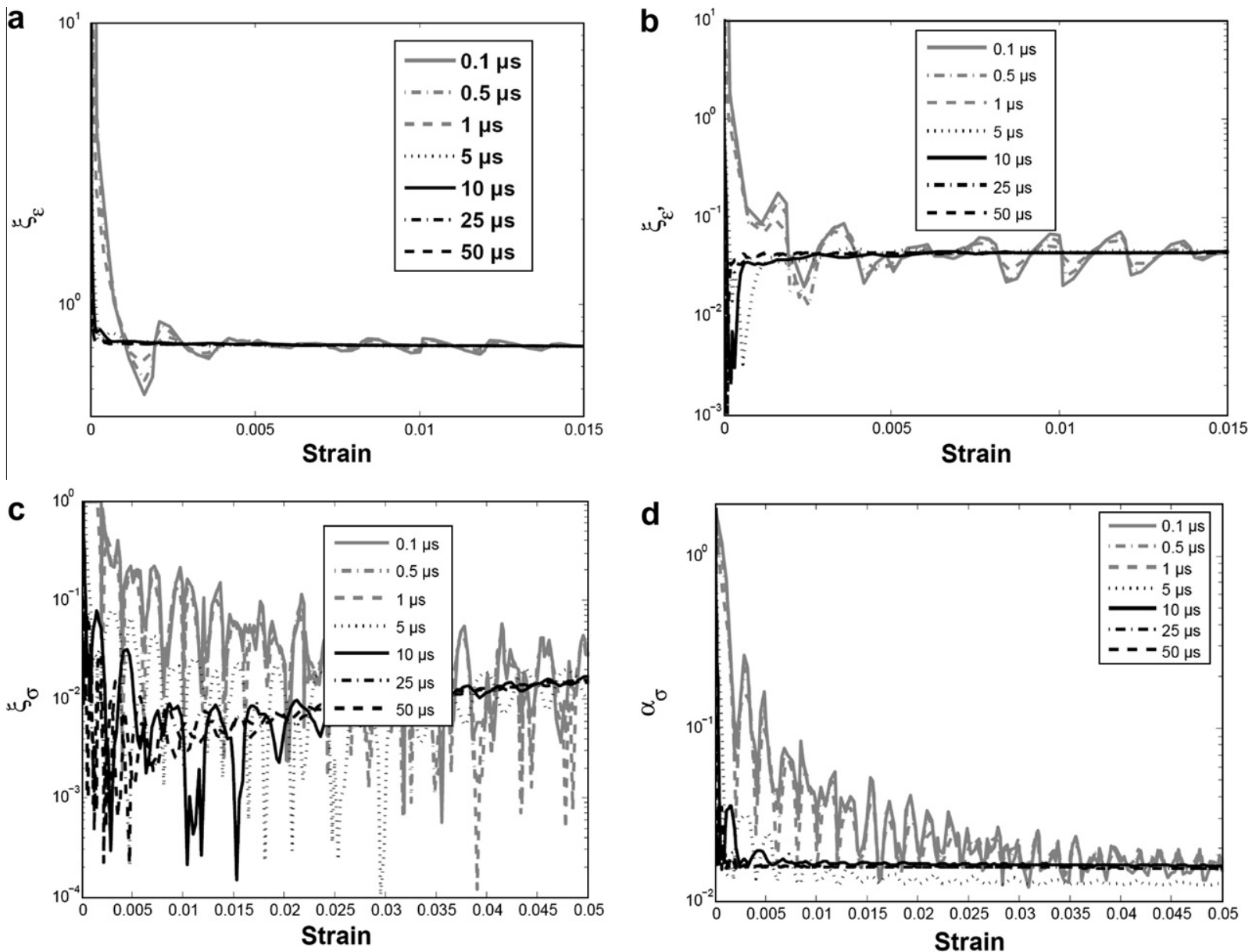

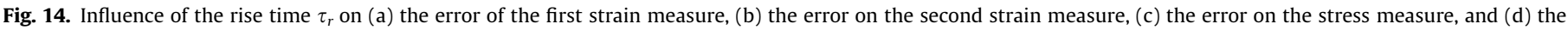
stress homogeneity coefficient. 


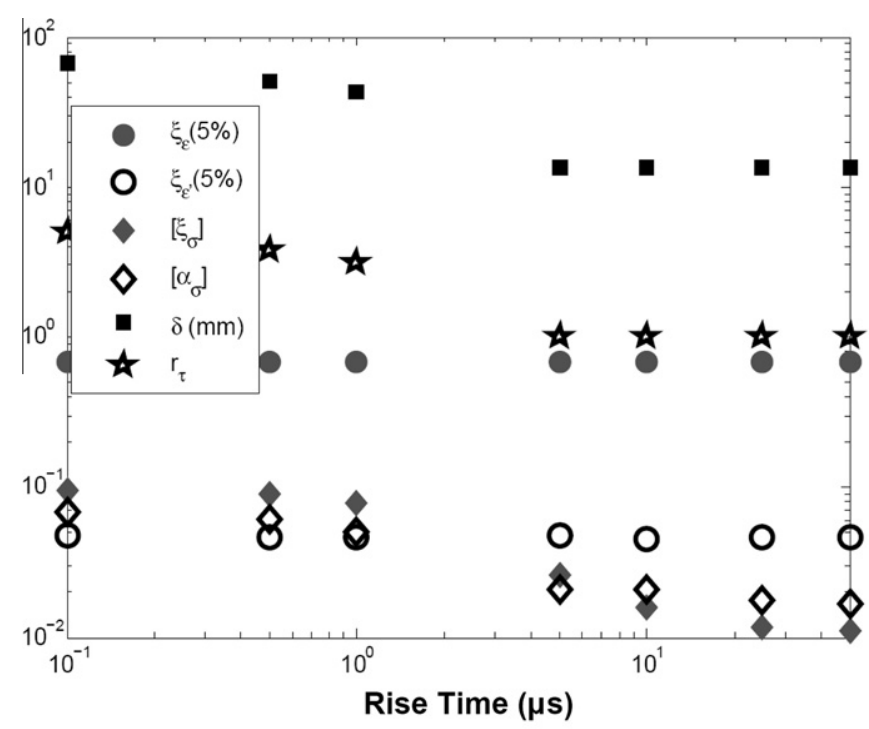

Fig. 15. Influence of the rise time $\tau_{r}$ on several parameters.

This corresponds to a nominal strain rate ranging from 100 to $10,000 \mathrm{~s}^{-1}$. The influence on the errors on the two strains measures is limited to the beginning of the test (see Fig. 16(a) and (b) and Fig. 17). On the other hand, the error on the stress increases with the velocity (see Fig. 16(c) and Fig. 17). Indeed, the stress homogenisation process occurs almost at the same time (see the variation of $\delta$ in Fig. 17). However, at high loading velocity (high strain rates) larger strains are achieved before the time $\tau_{h}$ (Fig. 16(d)). That is why, the error $\xi_{\sigma}$ increases with the loading velocity.

\section{Discussion}

In this section we will discuss separately the accuracy of strain and stress measurement.

\subsection{Strain measurement}

In this paper, we defined two strain measures. The first one, given by Eq. (5), is the conventional measure. It assumes that deformation only occurs at the central zone. Hence, only the length of this zone is considered in Eq. (5). The second measure is newly proposed in this paper and is given in Eq. (6). This measure assumes that the strain field is uniform in the total central and transition zones, consequently, the whole length of the central and transition zones in Eq. (6).

The errors on the two strain measures are decreasing in the first part of the numerical test. Subsequently, they take an almost constant value. The onsets of errors on the two strain measures are mainly threefold: (i) the dynamic induced strain field heterogeneity, (ii) the geometrical induced strain field heterogeneity, and (iii) the significance of deformation of transition zones.
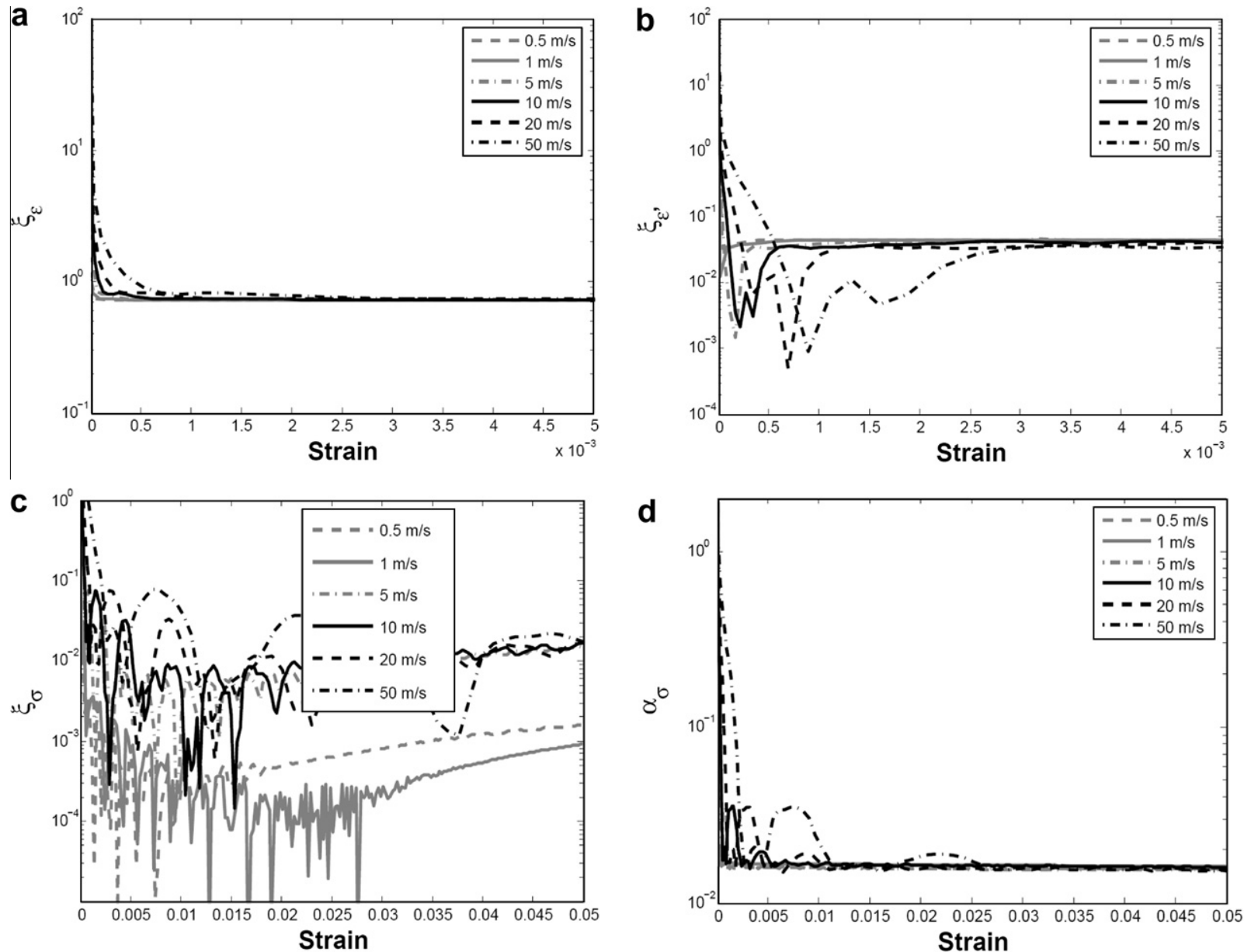

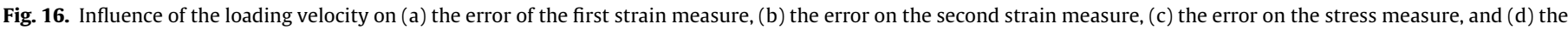
stress homogeneity coefficient. 


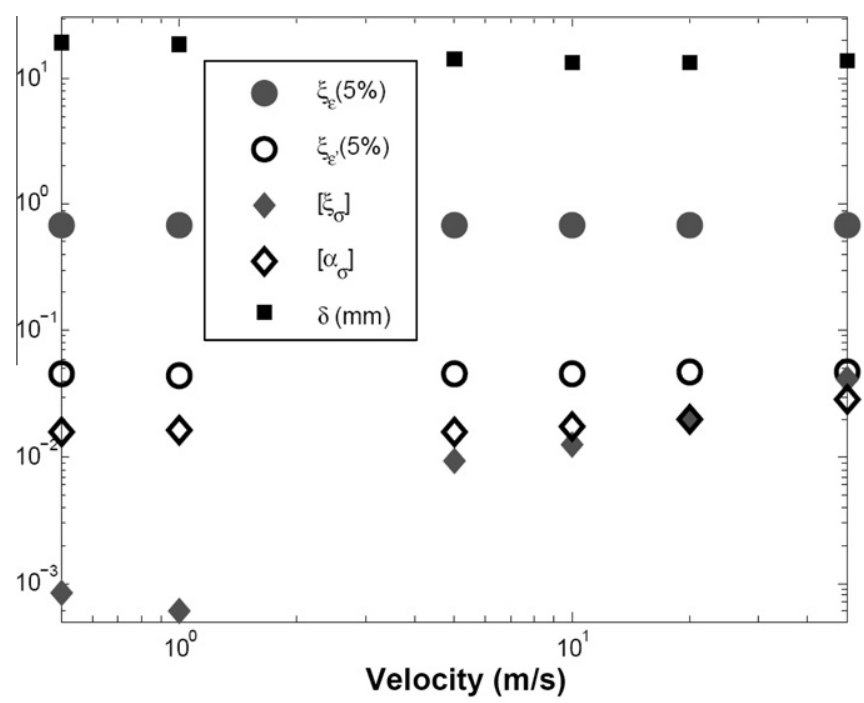

Fig. 17. Influence of the loading velocity on several parameters.

The dynamic induced strain field heterogeneity, which is due to wave propagation effects, is significant in the first transient part and disappears thereafter. Indeed, the second and third error onsets have an important influence in the second steady part of the test. These two onsets are mainly influenced by the geometrical parameters. That is why we observed a slight influence of the material and the testing parameters on the strain measures errors (see Sections 3.2 and 3.4).

The error $\xi_{\epsilon}$ on the first strain measure decreases with the length $l$ and increases with the radius $\rho$ and the width $w$. Therefore, we plot in Fig. $18 \xi_{\epsilon}$ in terms of a dimensionless coefficient:

$r=\frac{\rho \times w}{l^{2}}$.

$\xi_{\epsilon}$ is unsurprisingly increasing with $r$. An almost linear tendency is observed. Furthermore, the strain measure $\epsilon$ has always a bad accuracy. The best accuracy is obtained for $r=0.0032$. In this case, the error $\xi_{\epsilon}$ is $6.7 \%$.

Similarly to $\xi_{\epsilon}, \xi_{\epsilon^{\prime}}$ increases with the radius $\rho$ and decreases with the length $l$. However, $\xi_{\epsilon^{\prime}}$ decreases with the width $w$ contrar-

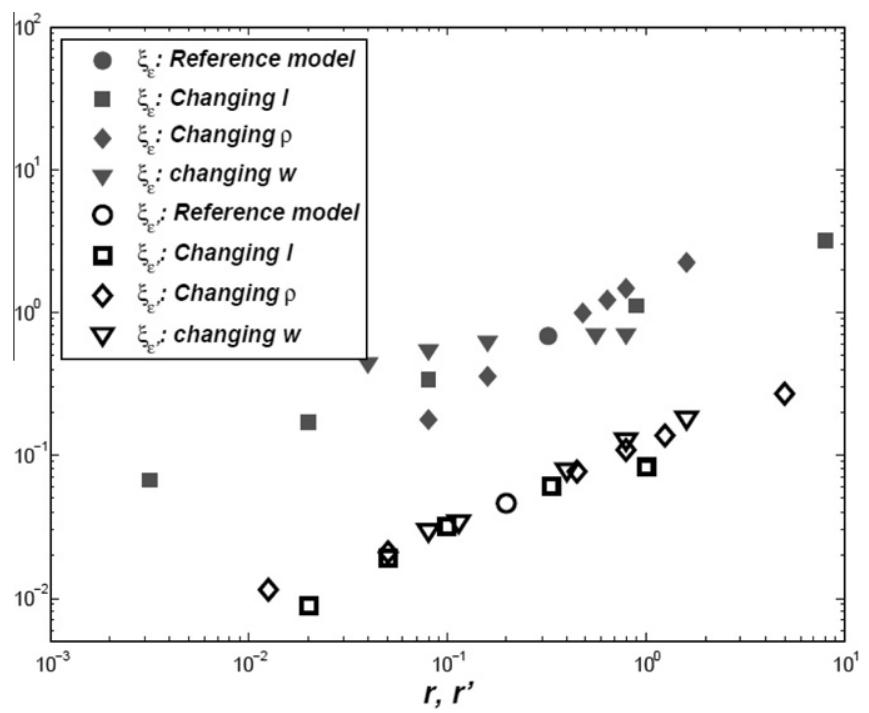

Fig. 18. Influence of $r$ and $r^{\prime}$ on $\xi_{\epsilon}$ and $\xi_{\epsilon^{\prime}}$, respectively. ly to $\xi_{\epsilon}$. Hence, we plot in Fig. $18 \xi_{\epsilon^{\prime}}$ in terms of a second dimensionless coefficient:

$r^{\prime}=\frac{\rho^{2}}{l \times w}$.

$\xi_{\epsilon^{\prime}}$ is increasing with $r^{\prime}$ as can be expected. A more pronounced linear behaviour can be observed. Moreover, the error $\xi_{\epsilon^{\prime}}$ ranges between $0.9 \%$ and $26.7 \%$. For the all studied cases, the accuracy of the second strain measure is always better than that of the first measure.

Regarding this study, we suggest that only the second measure should ne used. Furthermore, we propose to use geometries which fulfill the requirement:

$r^{\prime}<0.3$

Hence the error $\xi_{\epsilon^{\prime}}$ will be less than 0.05 (5\%).

\subsection{Stress measurement}

In this study, we considered the conventional stress measure, assuming that the applied load is instantly transmitted to any cross-section of the central zone. The error on the stress measure generally decreases, with time, while oscillating. The main error cause is the stress field heterogeneity which has two onsets: (i) dynamic induced heterogeneity, and (ii) geometrical induced heterogeneity. We note that the first onset is more important in the case of the stress measure.

Excepting the width $w$ of the central zone, the other studied parameters seems to have an influence on the stress measure accuracy. The rise time $\tau_{r}$ has a significant influence when it is lower than $\tau_{h}$, the time needed to reach stress homogeneity. The favorable case is $\tau_{r}>\tau_{h}$. Indeed, the error is almost constant and takes the lowest value. Assuming this case, we study, in Fig. 19, the variation of the error on the stress measure in terms of the dimensionless coefficient:

$r^{\prime \prime}=\frac{\rho+L}{\tau_{0} \times C_{0}}$.

The wave velocity $C_{0}$ and test duration $\tau_{0}$ are introduced to take into account the influence of the material and loading velocity, respectively. For the all studied cases, $\xi_{\sigma}$ is lower than $7 \%$. However, we recommend to choose the radius $\rho$ and the length $l$ such that

$r^{\prime \prime}<0.03$

in order to have an error on the stress measure lower than 0.05 (5\%).

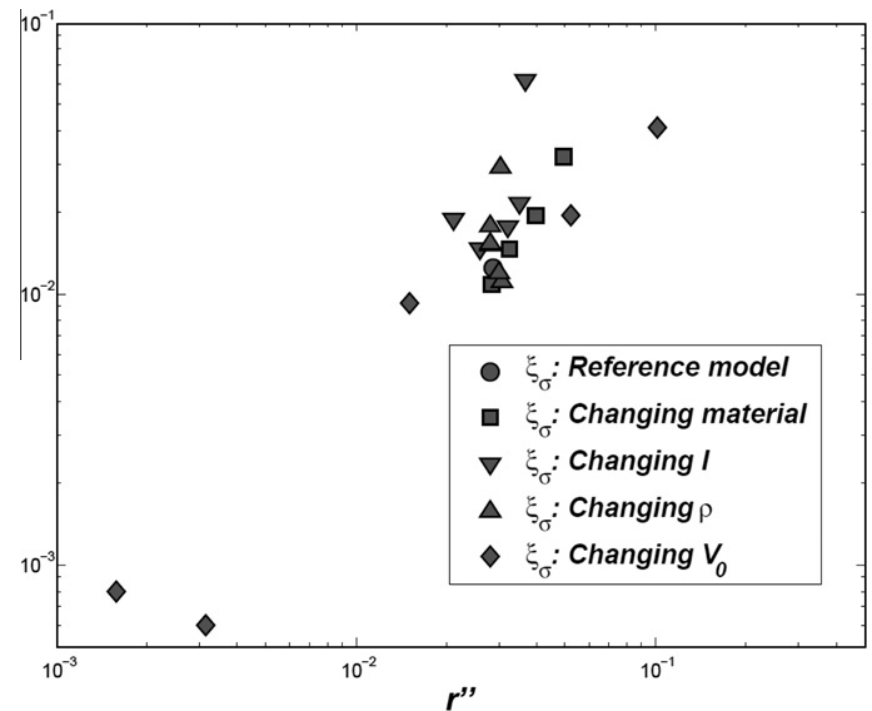

Fig. 19. Influence of $r^{\prime \prime}$ on $\xi_{\sigma}$. 


\section{Conclusion}

In this paper, we analysed the errors on strain and stress measurement in dynamic tensile experiments. For this purpose, we used the finite-element method. The influence of several parameters, on the strain and stress errors, is investigated. In this study, we considered two strain measures. The first one is conventional and the second is new. Both measures are significantly influenced by the sample geometry. Furthermore, the new proposed measure always give better results than the conventional one. As a first conclusion of this work, we gave a criterion to achieve error less than $5 \%$ on the second strain measure. On the other hand, we studied the error on the conventional stress measure. It comes that this error is highly correlated to the stress field homogeneity. Secondly, we suggest that the loading rise time should be higher than the time needed to achieve stress field homogeneity. In this framework, we gave an additional criterion to achieve an error less than $2 \%$ on the stress measure.

\section{References}

[1] Zhao H. Material behaviour characterisation using SHPB techniques, tests and simulations. Comput Struct 2003;81:1301-10.

[2] Kolsky H. An investigation of the mechanical properties of materials at very high rates of loading. Proc Phys Soc 1949;B62:676700.

[3] Tedesco JW, Hughes ML, Ross CA. Numerical simulation of high strain rate concrete compression tests. Comput Struct 1994;51:65-77.

[4] Othman R, Gary G. Testing aluminum alloy from quasi-static to dynamic strain-rates with a modified split Hopkinson bar method. Exp Mech 2007;47:295299.

[5] Benaceur I, Othman R, Guégan, Dhieb A, Damek F. Sensitivity of the flow stress of Nylon 6 and Nylon 66 to strain-rate. Int J Modern Phys B 2008:22:1249-54.

[6] Ogawa K. Mechanical behavior of metal under tension-compression loading at high strain rate. Int J Plast 1985;1:347-58.

[7] Lee OS, Kim MS. Dynamic material property characterisation by using split Hopkinson pressure bar (SHPB) technique. Nucl Eng Des 2003;226:119-25.

[8] Clark DS, Wood DS. The influence of specimen dimension and shape on the results in tension impact testing. Am Soc Mech Eng Counc Rep Membersh 1957:38:577-85.

[9] Staab GH, Gilat A. A direct-tension split Hopkinson bar for high strain-rate testing. Exp Mech 1991;31:232-5.

[10] Hughes ML, Tedesco JW, Ross CA. Numerical analysis of high strain rate splitting-tensile tests. Comput Struct 1993;47:653-71.

[11] Mouro P, Gary G, Zhao H. Dynamic tensile testing of sheet metal. J Phys IV France 2000;10:149-53.

[12] Huh H, Kang WJ, Han SS. A tension split Hopkinson bar for investigating the dynamic behavior of sheet metals. Exp Mech 2002;42:8-17.

[13] Mohr D, Gary G. M-shaped specimen for the high-strain rate tensile testing using a split Hopkinson pressure bar apparatus. Exp Mech 2007;47:681-92.
[14] Rusinek A, Cheriguene R, Bäumer, Klepaczko JR, Larour P. Dynamic behaviour of high-strength sheet steel in dynamic tension: experimental and numerical analyses. J Strain Anal Eng Des 2008;43:37-53.

[15] Othman R, Guégan P, Challita G, Pasco F, LeBreton D. A modified servohydraulic machine for testing at intermediate strain rates. Int J Impact Eng 2009;36:460467.

[16] Boyce BL, Dilmore MF. The dynamic tensile behavior of tough, ultrahighstrength steels at strain-rates from $0.0002 \mathrm{~s}^{-1}$ to $200 \mathrm{~s}^{-1}$. Int J Impact Eng 2009;36:263-71.

[17] Klepaczko JR, Nguyen HV, Nowacki WK. Quasi static and dynamic shearing of sheet metals. Eur J Mech - A/Solids 1999;18:271-89.

[18] Gilat A, Cheng CS. Torsional split Hopkinson bar tests at strain rates above 104 s-1. Exp Mech 2000;40:54-9.

[19] Rusinek A, Klepaczko JR. Shear testing of a sheet steel at wide range of strain rates and a constitutive relation with strain rate and temperature dependence of the flow stress. Int J Plast 2001;17:87-115.

[20] Challita G, Othman R, Guégan, Khalil K, Poitou A. New experimental sample for shear testing of adhesively bonded assemblies. Int J Modern Phys B 2008;22:1081-6.

[21] Miyauchi K. Stress strain relationship in simple shear of in-plane deformation for various steel sheets. Int J Mater Form 2008:233-6.

[22] Grolleau V, Gary G, Mohr D. Biaxial testing of sheet materials at high strain rates using viscoelastic bars. Exp Mech 2008;48:293306.

[23] Davies E, Hunter S. The dynamic compression testing of solids by the method of the split Hopkinson pressure bar. J Mech Phys Solids 1963;11:155-79.

[24] Gorham DA. Specimen Inertia in high strain rate compression. J Phys D Appl Phys 1989;22:1888-93.

[25] Gorham DA. The effect of specimen dimensions on high strain rate compression measurements of copper. J Phys D Appl Phys 1991;24:1489-92.

[26] Dioh NN, Leevers PS, Williams JG. Thickness effects in split Hopkinson pressure bar tests. Polymer 1993;34:4230-4.

[27] Zhao H. A study on specimen thickness effects in the impact tests on polymers by numeric simulations. Polymer 1998:39:1102-206.

[28] Verleysen P, Degrieck J. Non-homogeneous and multi-axial stress distribution in concrete specimens during split Hopkinson tensile tests. Comput Struct 2000;77:669-76.

[29] Lee OS, Kim GH. Thickness effects on mechanical behavior of a composite material (1001P) and polycarbonate in split Hopkinson pressure bar technique. J Mater Sci Lett 2000;19:1805-8.

[30] Meng H, Li QM. Correlation between the accuracy of a SHPB test and the stress uniformity based on numerical experiments. Int J Impact Eng 2003;28:537-55.

[31] Song B, Chen W. Dynamic stress equilibration in split Hopkinson pressure bar tests on soft materials. Exp Mech 2004;44:300312.

[32] Aloui S, Othman R, Poitou A, Guégan P, El-Borgi S. Non-parametric identification of the non-homogeneous stress in high strain-rate uni-axial experiments. Mech Res Commun 2008;35:392397.

[33] Verleysen P, Degrieck J, Verstraete T, Van Slycken J. Influence of specimen geometry on split Hopkinson tensile bar tests on sheet materials. Exp Mech 2008;48:587-98.

[34] Challita G, Othman R. Finite-element analysis of SHPB tests on double-lap adhesive joints. Int J Adhes Adhes 2010;30:236-44.

[35] Maringa M. Dimensioning of dog bone specimens and numerical analysis of the effects of different fillet radii, clamp area and pinhole loading on the stresses in such specimens. African J Sci Technol Sci Eng Ser 2004;5:60-72. 